University of Nebraska - Lincoln

DigitalCommons@University of Nebraska - Lincoln

August 2007

\title{
Scaling Behavior of the Exchange-Bias Training Effect
}

\author{
Christian Binek \\ University of Nebraska-Lincoln, cbinek@unl.edu \\ Srinivas Polisetty \\ University of Nebraska-Lincoln, polisetty.srinivas@gmail.com \\ Sarbeswar Sahoo \\ University of Nebraska-Lincoln, sarbeswar@gmail.com
}

Follow this and additional works at: https://digitalcommons.unl.edu/physicsbinek

Part of the Physics Commons

Binek, Christian; Polisetty, Srinivas; and Sahoo, Sarbeswar, "Scaling Behavior of the Exchange-Bias Training Effect" (2007). Christian Binek Publications. 56.

https://digitalcommons.unl.edu/physicsbinek/56

This Article is brought to you for free and open access by the Research Papers in Physics and Astronomy at DigitalCommons@University of Nebraska - Lincoln. It has been accepted for inclusion in Christian Binek Publications by an authorized administrator of DigitalCommons@University of Nebraska - Lincoln. 


\title{
Scaling behavior of the exchange-bias training effect
}

\author{
Srinivas Polisetty, Sarbeswar Sahoo, and Christian Binek* \\ Department of Physics and Astronomy and the Nebraska Center for Materials and Nanoscience, \\ University of Nebraska-Lincoln, Lincoln, Nebraska 68588-0111, USA
}

(Received 27 August 2007; published 19 November 2007)

\begin{abstract}
The dependence of the exchange-bias training effect on temperature and ferromagnetic film thickness is studied in detail and scaling behavior of the data is presented. Thickness-dependent exchange bias and its training are measured using the magneto-optical Kerr effect. A focused laser beam is scanned across a Co wedge probing local hysteresis loops of the $\mathrm{Co}$ film which is pinned by an antiferromagnetic $\mathrm{CoO}$ layer of uniform thickness. A phenomenological theory is best fitted to the exchange-bias training data resembling the evolution of the exchange-bias field on subsequently cycled hysteresis loops. Best fits are done for various temperatures and Co thicknesses. Data collapse on respective master curves is achieved for the thickness and temperature-dependent fitting parameters as well as the exchange bias and coercive fields of the initial hysteresis loops. The scaling behavior is strong evidence for the validity and the universality of the underlying theoretical approach based on triggered relaxation of the pinning layer towards quasiequilibrium.
\end{abstract}

DOI: 10.1103/PhysRevB.76.184423

PACS number(s): 75.60.- d, 75.70.Cn

\section{INTRODUCTION}

Proximity and size effects are cornerstones of modern condensed matter physics. ${ }^{1-4}$ Exchange bias (EB) and its accompanying training effect represent a magnetic proximity phenomenon which takes place at the interface of exchange coupled ferromagnetic (FM) and antiferromagnetic (AF) heterostructures. ${ }^{5-10}$ In the proximity of an AF pinning layer a FM film can experience an exchange induced unidirectional anisotropy. The latter reflects its presence by a shift of the FM hysteresis along the magnetic field axis and is quantified by the amount $\mu_{0} H_{\mathrm{EB}}$. The EB effect is initialized by field cooling the heterosystem to below the blocking temperature $T_{B}$, where $\mathrm{AF}$ order establishes at least on mesoscopic scales. ${ }^{11}$ The shift of the FM hysteresis loop along the magnetic field axis is often accompanied by an EB induced loop broadening. ${ }^{12,13}$ In addition, a gradual degradation of the EB field can take place when cycling the heterostructure through consecutive hysteresis loops. ${ }^{14-21}$ This aging phenomenon is known as training effect and is quantified by the $\mu_{0} H_{\mathrm{EB}}$ vs $n$ dependence, where $n$ labels the number of loops cycled after initializing the EB via field cooling. EB and the accompanying training effect have been observed in various magnetic systems. ${ }^{7,22-24}$

Recently size effects involved in the EB phenomenon have been extensively studied. ${ }^{25-28}$ This includes the dependence of the EB on the AF and FM film thicknesses as well as size effects induced by lateral structuring of the FM and AF components of EB heterostructures. Various characteristic length scales influencing the EB have been identified. For instance, finite $\mathrm{AF}$ anisotropy gives rise to a critical thickness $t_{\mathrm{AF}}$ of the pinning layer below which EB disappears. ${ }^{5,6,29-31}$ Moreover, lateral structuring on a scale comparable with $\mathrm{AF}$ and FM domain sizes and domain wall widths affects the characteristics of the EB.

The most frequently studied size effect in EB systems is given by the $1 / t_{\mathrm{FM}}$ thickness dependence of the EB field on the FM film thickness $t_{\mathrm{FM}} \cdot{ }^{27,28,32-34}$ The inverse FM thickness dependence reveals the interface nature of the EB effect and reflects the origin of $\mathrm{EB}$ as a competition between the Zeeman energy of the FM layer and AF/FM interface coupling energy. It is the detailed microscopic understanding of the latter which is still under debate. However, under the assumption of homogeneous magnetization along the FM film normal, the Zeeman energy will increase linearly with $t_{\mathrm{FM}}$ independent of the specific nature of the interface coupling energy.

This manuscript sheds light on the $t_{\mathrm{FM}}$ dependence of the EB training effect and, in particular, its scaling behavior. Training, which describes the decrease of the EB field with subsequently cycled hysteresis loops of the ferromagnet, can be understood in the framework of triggered spin configurational relaxation of the AF pinning layer. This general view includes deviations of the AF spins from their easy axes and, hence, from the AF ground state of the pinning layer. Recently such deviations and reorientations of spins between easy axes have been evidenced as a microscopic origin for large training effects and asymmetry in EB systems such as $\mathrm{CoO} / \mathrm{Co}$ where more than one easy axis exists. ${ }^{18,35,36}$

Since in this general sense training originates from changes of the spin structure of the pinning layer towards its equilibrium configuration, it is not apparent that a variation of the FM thickness could at all affect the EB training effect. A closer look reveals, however, the need of studying the FM thickness dependence of the EB training effect.

EB is an interface phenomenon and the EB fields may follow a $\mu_{0} H_{\mathrm{EB}} \propto 1 / t_{\mathrm{FM}}$ dependence. If this simple $1 / t_{\mathrm{FM}}$ dependence holds for each individual hysteresis loop of a training sequence according to $\mu_{0} H_{\mathrm{EB}}(n) \propto 1 / t_{\mathrm{FM}}$ then one can conclude that the $n$-dependent evolution of the AF interface magnetization is independent of $t_{\mathrm{FM}}$. Note, that such a finding is not apparent considering the fact the antiferromagnet acts on the ferromagnet by changing its coercivity and a counter-reaction of some sort has to be expected. ${ }^{37,38}$ In addition, even the simple $1 / t_{\mathrm{FM}}$ dependence of $\mu_{0} H_{\mathrm{EB}}(n)$ leaves a nontrivial fingerprint in the characteristics of the training sequence allowing for a unique cross-check of the recently introduced theoretical approach. ${ }^{39,40}$ Scaling of the 


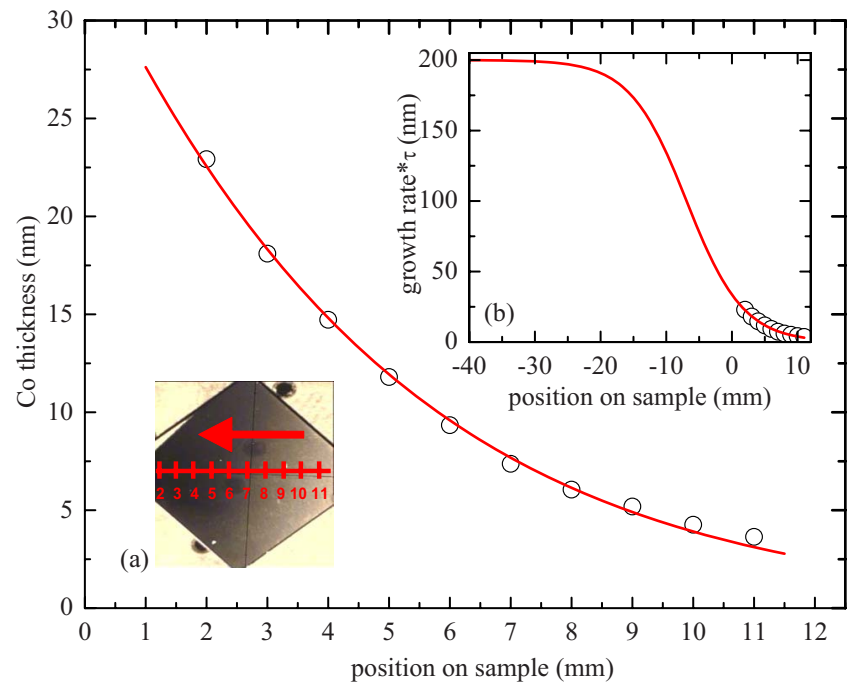

FIG. 1. (Color online) Variation of Co thickness with respect to the position $x$ parallel to the thickness gradient. Local thicknesses are obtained from x-ray reflectivity (circles). An empirical Fermitype function is best fitted to the data (line). Inset (a) shows an optical micrograph of the wedge sample. The arrow points in the direction of the thickness gradient. The scale defines the positions on the sample. Inset (b) shows an extrapolation of the empirical Fermi-type flux profile created by the partially shuttered evaporation beam (line) along with the data points (circles).

crucial parameter involved in the fits of the $\mu_{0} H_{\mathrm{EB}}$ vs $n$ data and its collapse on a thickness and temperature-dependent master curve provides hitherto unprecedented evidence for the universality of our phenomenological description of the EB training effect.

\section{SAMPLE PREPARATION AND EXPERIMENTAL DETAILS}

We use molecular beam epitaxy (MBE) technique to grow a wedge shaped epitaxial Co thin film on the $c$ plane $\mathrm{Al}_{2} \mathrm{O}_{3}$ substrate. Deposition takes place under ultrahigh vacuum (UHV) condition at a base pressure of $5.0 \times 10^{-11} \mathrm{mbar}$ and a substrate temperature of $573 \mathrm{~K}$. An average thickness gradient of 3 to $28 \mathrm{~nm}$ over $1 \mathrm{~cm}$ lateral distance was achieved by partially opening the shutter of the effusion cell and projecting the truncated beam profile onto the substrate. Unlike other step wedges where sample growth was controlled by using shutter motion attached to the substrate, ${ }^{32,33,41}$ we exploit shutter control of the Co effusion cell allowing for the growth of a continuous Co wedge.

Figure 1 shows the local Co thickness probed along the direction of the thickness gradient at individual positions $x$ of the wedge. Inset (a) of Fig. 1 shows an optical micrograph of the sample revealing the lateral change of optical transparency and hence, resembling the thickness gradient of the wedge. The latter is indicated by an arrow.

Local thicknesses have been measured by small angle $\mathrm{x}$-ray reflectivity (XRR) using collimated $\mathrm{x}$ rays with a lateral resolution of about $\delta x \approx 0.5 \mathrm{~mm}$ in the direction of the gradient while the grazing incidence of the $\mathrm{x}$ rays gives rise
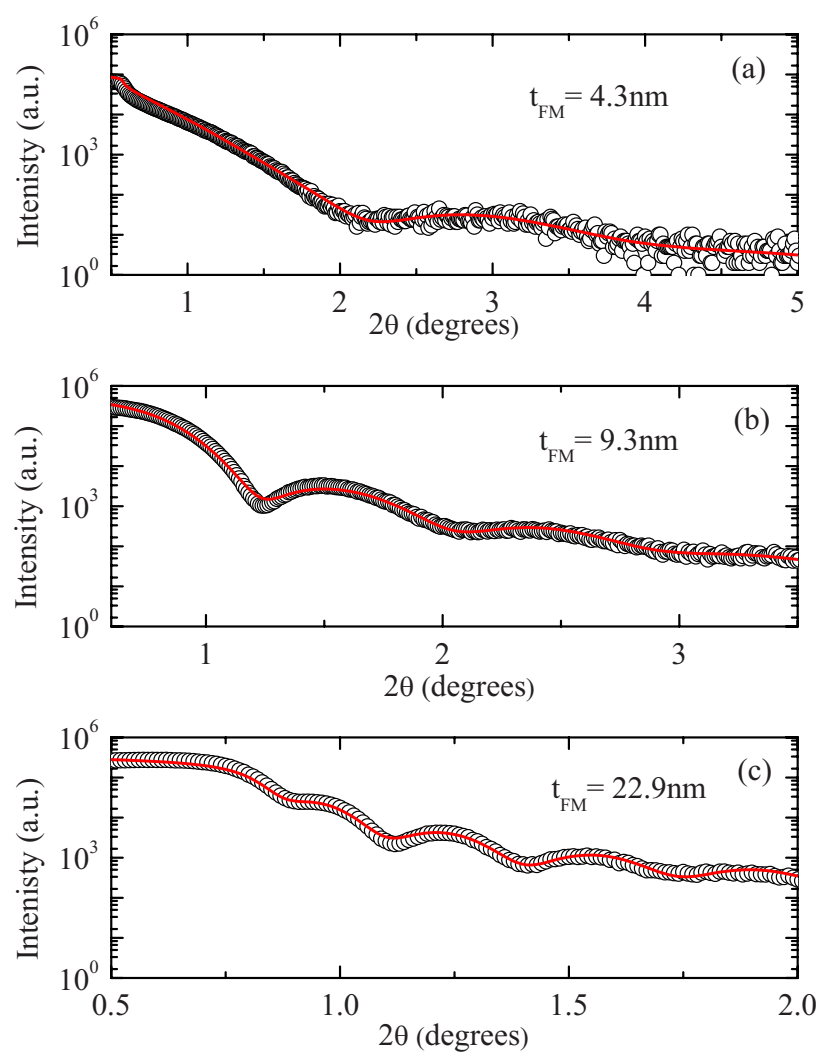

FIG. 2. (Color online) Small angle x-ray reflectivity data (circles) for three different Co thicknesses (a) $4.3 \mathrm{~nm}$, (b) $9.3 \mathrm{~nm}$, and (c) $22.9 \mathrm{~nm}$ obtained from best fits (lines) using the LEPTOS-2 software package.

to a spatial average normal to the gradient. Note that this direction is expected to be of constant Co thickness in accordance with the growth technique. Figures 2(a)-2(c) show three typical XRR $\theta-2 \theta$ scans taken at different positions. Best fits (lines) reveal the thicknesses $t_{\mathrm{FM}}\left(x_{1}=10 \mathrm{~mm}\right)$ $=4.3 \mathrm{~nm}, \quad t_{\mathrm{FM}}\left(x_{2}=6 \mathrm{~mm}\right)=9.3 \mathrm{~nm}, \quad$ and $\quad t_{\mathrm{FM}}\left(x_{3}=2 \mathrm{~mm}\right)$ $=22.9 \mathrm{~nm}$.

Since the wedge resembles the projected flux profile of the partially closed Co effusion cell onto the sapphire substrate, the local Co thickness is a nonlinear function of the lateral position $x$. In order to obtain a quantitative relation $t_{\mathrm{FM}}=t_{\mathrm{FM}}(x)$ which allows for continuous thickness interpolation, the locally measured thickness data are fitted to an empirical profile $t(x)$. The latter has been modeled with the help of a Fermi-type function $t(x)=A /\left(e^{\left(x-x_{0}\right) / w}+1\right)$. It is an empirical approach replacing the cosine law of ideal pointlike Knudsen cells where constant flux is realized on spherical surfaces touching the evaporation point. ${ }^{42}$ Here, however, we take advantage of the perturbation of the flux induced by a shutter. Collision of Co atoms leaving the cell gives rise to momentum transfer and, hence, to a broadening of the otherwise geometrically sharp shadow. The broadening is modeled by the width $w$ entering the profile function $t(x)$. The unperturbed Co evaporation rate in the center of the flux profile was monitored by a calibrated quartz crystal and found to be $2 t\left(x_{0}\right) / \tau=0.02 \mathrm{~nm} / \mathrm{s}$. The sapphire substrate has been exposed to the Co evaporation profile for $\tau=10^{4} \mathrm{~s}$ cali- 


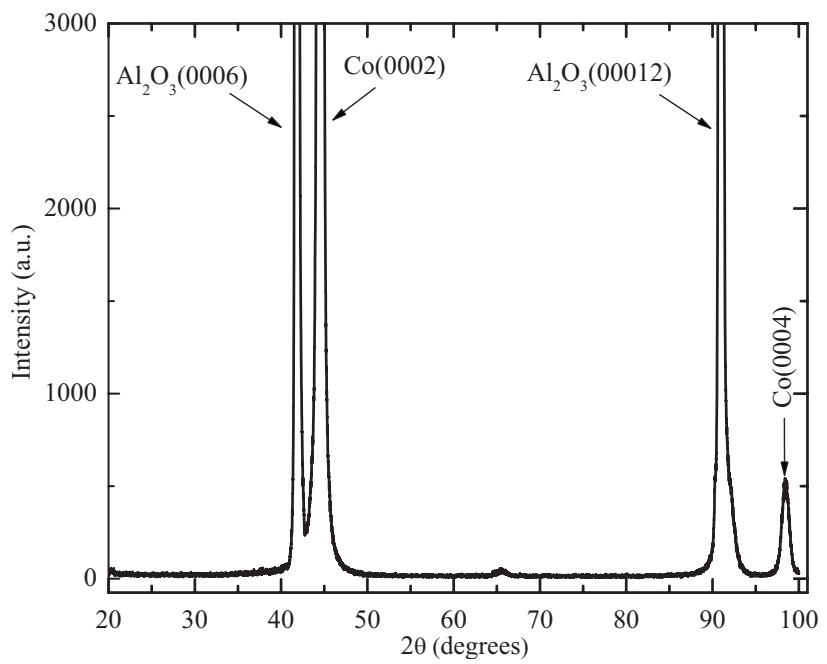

FIG. 3. X-ray diffraction pattern of the $\mathrm{Co} / \mathrm{CoO}$ film on $c-\mathrm{Al}_{2} \mathrm{O}_{3}$ substrate. Single-crystalline peaks of hexagonal Co film are identified.

brating $A=2 t\left(x_{0}\right)$ as $A=200 \mathrm{~nm}$. The two remaining parameters $x_{0}$ and $w$ adjust the onset and steepness of the flux drop from maximum unperturbed flux down to zero flux for $x$ $\gg x_{0}$. Best fitting yields $x_{0}=-6.91 \mathrm{~mm}$ and $w=4.32 \mathrm{~mm}$. The result of the best fit is displayed as a line in Fig. 1 and an enlarged scale in inset (b) of Fig. 1, respectively.

A naturally formed AF $\mathrm{CoO}$ layer of thickness $t_{\mathrm{AF}}$ $\approx 3 \mathrm{~nm}$ has been identified by small angle XRR after atmospheric exposure of the Co wedge at various positions along the wedge. The use of a single Co wedge ensures that the $\mathrm{CoO}$ pinning layer has constant thickness while $t_{\mathrm{FM}}$ varies continuously. This has advantages over the preparation of a sequence of individual samples with various Co thicknesses, because exposure time and various other ill controlled factors influence the thickness of the naturally formed $\mathrm{CoO}$ layer. Since we study the $t_{\mathrm{FM}}$ dependence of the $\mathrm{EB}$ and its training effect, a constant AF pinning layer thickness is crucial in order to avoid fluctuations in $\mu_{0} H_{\mathrm{EB}}$ induced by fluctuations in $t_{\mathrm{AF}}$. Note that in a wedge sample the local magnetization reversal can be affected by the neighboring FM parts of different thickness. Ideal studies may therefore favor a series of $\mathrm{Co} / \mathrm{CoO}$ bilayers with varying $\mathrm{Co}$ and constant $\mathrm{CoO}$ thickness similar to a sample series with constant $t_{\mathrm{FM}}$ and varying AF thickness, recently studied in Ref. 43. However, CoO grown by ex situ oxidization of the top Co layer does not guarantee reproducible AF film thicknesses throughout the individual samples. This is our major motivation for the wedge samples. In addition it is reasonable and experimentally evidenced that local EB effects on the length scale of the AF domains are virtually unaffected by their neighboring counterparts. ${ }^{11}$

Detailed structural characterization of the wedge $\mathrm{Co} / \mathrm{CoO}$ sample has been performed by $\theta-2 \theta$ wide angle $\mathrm{x}$-ray diffraction (XRD) and pole figure scans using the $\mathrm{Cu}-K_{\alpha}$ source of Rigaku D/Max-B diffractometer and Bruker-AXS D8, respectively. The XRD pattern of Fig. 3 reveals a singlecrystalline hexagonal Co film with (0002)-oriented growth on the $c-\mathrm{Al}_{2} \mathrm{O}_{3}$ substrate similar to the results found from

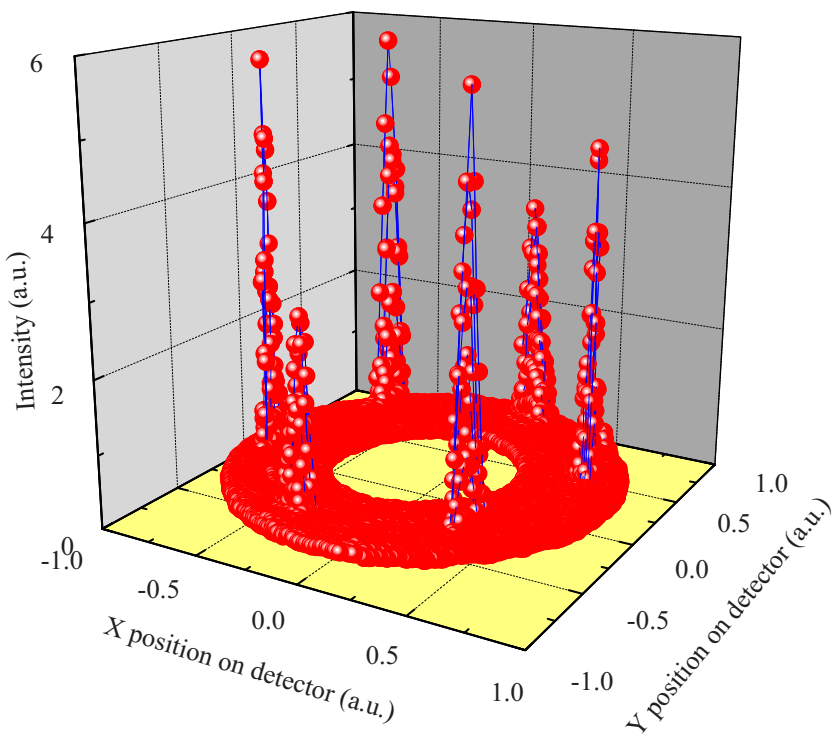

FIG. 4. (Color online) 3D pole figure scan performed at $2 \theta$ $=46.8^{\circ}$. Peak intensities separated by $60^{\circ}$ confirm the hexagonal in-plane symmetry of the Co film.

deposition on the $\alpha$ plane of sapphire in Ref. 44. The corresponding pole figure scan in Fig. 4 evidences the sixfold symmetry of the Co film confirming epitaxial hexagonal growth. The pole figure scans were performed at various Co thicknesses along the wedge keeping $2 \theta=44.2^{\circ}$ of $\mathrm{Co}(0002)$ fixed using the 2D detector (HI-STAR). They all reveal identical hexagonal symmetry.

\section{MAGNETIC CHARACTERIZATION}

We use the longitudinal magneto-optical Kerr effect (LMOKE) to measure the local magnetic hysteresis loops. Magnetic fields $-0.25 T \leqslant \mu_{0} H \leqslant 0.25 T$ are applied parallel to the sample surface. LMOKE loops were recorded at various temperatures $20 \mathrm{~K} \leqslant T \leqslant 70 \mathrm{~K}$ after cooling the sample from $T=320 \mathrm{~K}$ in the presence of a magnetic field of $0.25 \mathrm{~T}$. The $s$-polarized incident laser beam of wavelength $\lambda=670 \mathrm{~nm}$ makes an angel of about $20^{\circ}$ with the normal of the sample surface. Glan-Thompson polarizers are used for polarizing and analyzing of the light. A lens of focal length $f$ $=350 \mathrm{~mm}$ and diameter of $D=25 \mathrm{~mm}$ focuses the light beam onto the sample surface. The reflected beam is periodically modulated between left and right circularly polarized light by the photoelastic modulator (PEM). Modulation takes place with a frequency of $50 \mathrm{kHz}$ and a phase amplitude of $\varphi_{0}=175^{\circ}$ which maximizes the Bessel function $J_{2}(\varphi)$. The modulation signal is used as reference signal for a lock-in amplifier. The orthogonal retarder axes of the PEM are perpendicular and parallel aligned to the plane of incidence, respectively. The subsequent analyzer makes an angle of $45^{\circ}$ to the retarder axes. The transmitted intensity modulated light is detected by a photodiode providing the input signal to the lock-in amplifier. Its second harmonic Fourier component is proportional to the off-diagonal Fresnel reflection coefficient $r_{\mathrm{sp}}$ and, hence, proportional to the magnetization of 

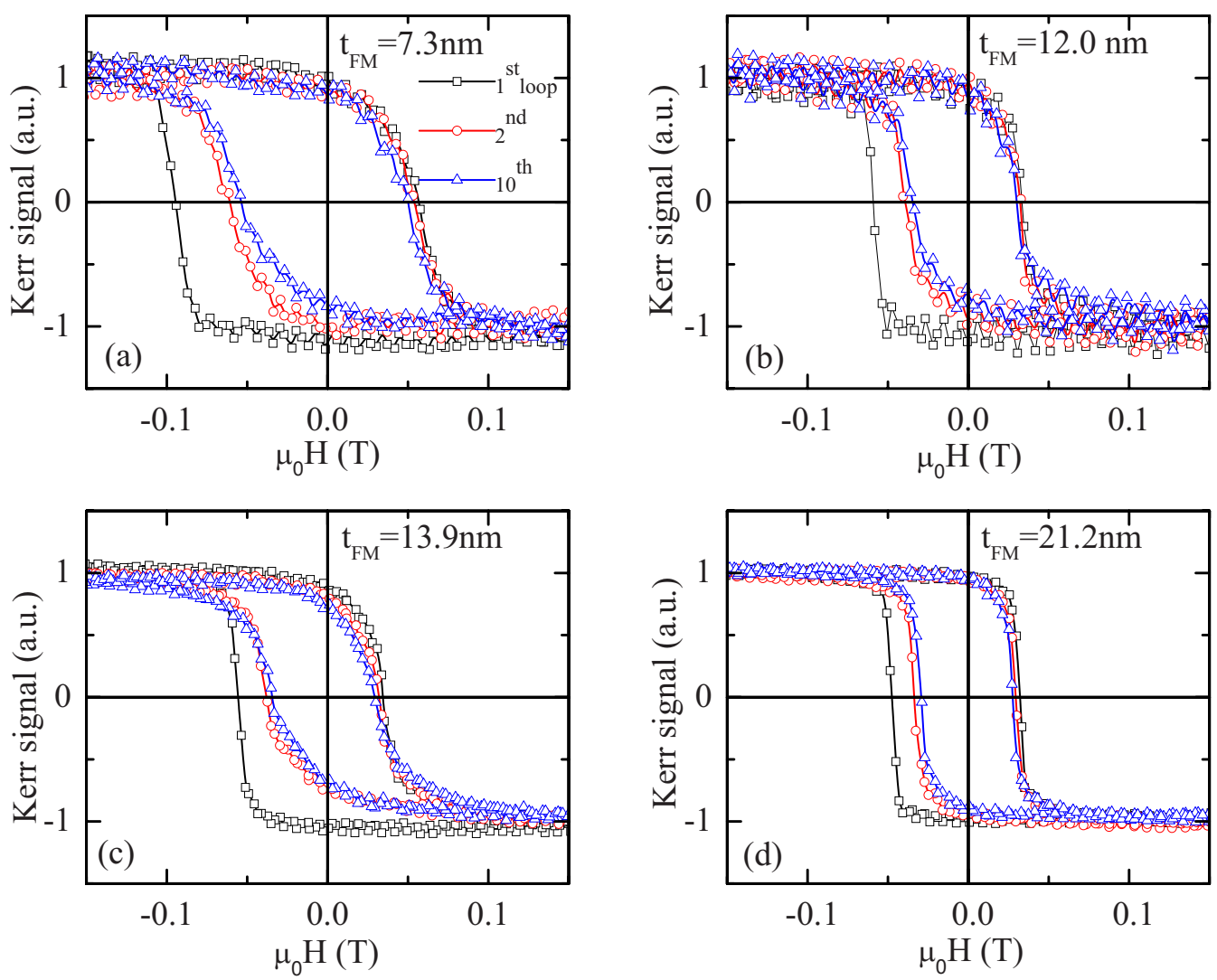

FIG. 5. (Color online) Normalized Kerr magnetic hysteresis loops measured at $T=50 \mathrm{~K}$ within a training sequence: first loop (squares), second loop (circles), and tenth loop (triangles) for four different Co thicknesses (a) $7.3 \mathrm{~nm}$, (b) $12.0 \mathrm{~nm}$, (c) $13.9 \mathrm{~nm}$, and (d) $21.2 \mathrm{~nm}$.

the sample within the penetration depth of the light beam. ${ }^{45}$

The focused laser beam is scanned across the wedge shaped Co film probing local hysteresis loops. The scan takes place parallel to the thickness gradient. The local thickness is identified from readings of the respective laser spot positions on an mm scale attached to the sample. The diameter of the laser spot is diffraction limited according to the Rayleigh criterion $\Delta l=1.22 f \lambda / D \approx 11 \mu \mathrm{m}$. Taking into account the limited spatial resolution of the x-ray beam as well as reading errors in the local laser spot position due to parallax, outshining of the Airy disk and inaccuracy in the scale attached to the sample we estimate a total uncertainty in the position reading to be $\Delta x<1 \mathrm{~mm}$. This uncertainty gives rise to a relative thickness uncertainty. With $x_{0}=-6.91 \mathrm{~mm}$ and $w=4.32 \mathrm{~mm}, e^{\left(x-x_{0}\right) / w} \gg 1$ holds for all positions $2 \mathrm{~mm}<x$ $<11 \mathrm{~mm}$ and, hence, $\Delta t / t$ is estimated according to $\Delta t / t$ $=|\partial t / \partial x| \Delta x / t \approx \Delta x / w \lesssim 23 \%$. However, the uncertainty in the Co thickness is corrected to large extends with the help of the scaling plots as outlined subsequently.

\section{RESULTS AND DISCUSSION}

The investigation of the EB training effect requires the standard initialization of the EB prior to every set of subsequently cycled hysteresis loops. A well defined EB initialization takes place via field cooling the sample from $T$ $=320 \mathrm{~K}>T_{N}(\mathrm{CoO})=291 \mathrm{~K}$ to $T=20 \mathrm{~K}$ in the presence of an in-plane applied magnetic field of $\mu_{0} H=0.25 \mathrm{~T}$. The latter exceeds the saturation field of our Co wedge. Note, that the easy axis of Co films with thicknesses $3 \mathrm{~nm}<t_{\mathrm{FM}}<28 \mathrm{~nm}$ is in-plane ${ }^{46-48}$ while the variation of the in-plane anisotropy expected from the structural sixfold symmetry of Co (0002) has negligible impact on the hysteresis loops. After EB initialization a fixed temperature between $20 \mathrm{~K}<T<T_{B}$ $=96.8 \mathrm{~K}$ is stabilized with $\delta T<10 \mathrm{mK}$ precision in a closed cycle optical cryostat (Janis Research CCS-350SH). Measurements of the local training effect were preformed at a fixed position $x$ by recording subsequently cycled longitudinal Kerr loops in a field interval $-0.25 \mathrm{~T}<\mu_{0} H<0.25 \mathrm{~T}$. The EB shift $\mu_{0} H_{\mathrm{EB}}=\mu_{0}\left(H_{c 1}+H_{c 2}\right) / 2$ of the hysteresis loop is determined for each individual loop from the coercive fields $H_{c_{1,2}}$ by linear best fits in the vicinity of zero magnetization $M\left(H_{c_{1}}\right)=M\left(H_{c_{2}}\right)=0$.

Figures 5(a)-5(d) show the hysteresis of the first (squares), second (circles), and tenth (triangles) loops for $\mathrm{CoO}(\sim 3 \mathrm{~nm}) / \mathrm{Co}\left(t_{\mathrm{FM}}\right)$. Measurements take place at various positions corresponding to the nominal thicknesses $t_{\mathrm{FM}}=7.3$, $12.0,13.9$, and $21.2 \mathrm{~nm}$ at $T=50 \mathrm{~K}$ after $\mathrm{EB}$ initialization, respectively. A pronounced $\mathrm{EB}$ and $\mathrm{EB}$ training effect accompanied by a change in the loop width $\mu_{0} H_{c}=\mu_{0}\left(H_{c 2}\right.$ $-H_{c 1}$ ) is shown. Typically $\sim 80 \%$ of the training dynamics takes place between the first and second loop while the remaining $20 \%$ decay gradually with increasing number of loops. Figures 6(a)-6(d) show $\mu_{0} H_{\mathrm{EB}}$ vs $n$ at $T=50 \mathrm{~K}$ for all nominal thicknesses. Circles are the experimental data while squares are obtained from the best fit of the theory discussed 

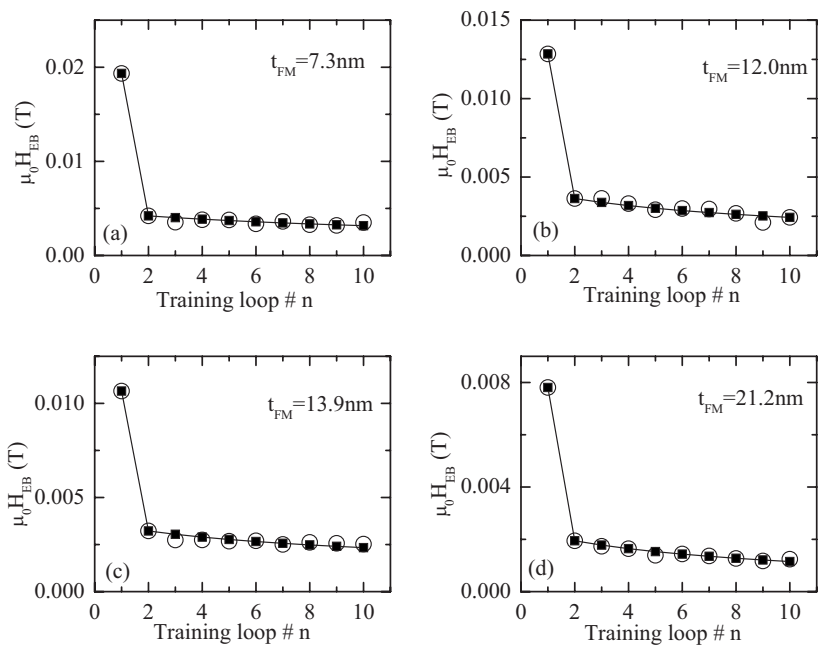

FIG. 6. Training effect of the exchange bias $\mu_{0} H_{\mathrm{EB}}$ vs loop No. $n$ (circles) and the corresponding best fits according to Eq. (3) (squares) for the same Co thicknesses as displayed in Fig. 5 measured at $T=50 \mathrm{~K}$. Lines are guide to the eye only.

below. In addition to the displayed data, training sequences of 10 subsequent loops have been measured and best fitted for the nominal Co thicknesses $t_{\mathrm{FM}}=7.3,12.0,13.9$, and $21.2 \mathrm{~nm}$ at various temperatures $T=20,27,35,43,50,57$, 65 , and $70 \mathrm{~K}$, respectively.

Figure 7(a) shows the EB fields $\mu_{0} H_{\mathrm{EB}}(n=1)$ vs $T$ of the first loop of a respective training sequence for all measured thicknesses $t_{\mathrm{FM}}$ and temperatures $T$. Apparently, but in the absence of a proper theory, the individual data sets $\mu_{0} H_{\mathrm{EB}}$ $\left(n=1, \quad t_{\mathrm{FM}}=7.3 \mathrm{~nm}\right)$ vs $T$ (squares), $\mu_{0} H_{\mathrm{EB}} \quad\left(n=1, \quad t_{\mathrm{FM}}\right.$ $=12.0 \mathrm{~nm})$ vs $T$ (circles), $\mu_{0} H_{\mathrm{EB}}\left(n=1, t_{\mathrm{FM}}=13.9 \mathrm{~nm}\right)$ vs $T$ (up triangles), and $\mu_{0} H_{\mathrm{EB}}\left(n=1, t_{\mathrm{FM}}=21.2 \mathrm{~nm}\right)$ vs $T$ (down triangles) follow a linear temperature dependence, respectively. The lines are linear best fits to the data.

In accordance with the Meiklejon Bean expression

$$
\mu_{0} H_{\mathrm{EB}}=-\frac{J S_{\mathrm{FM}} S_{\mathrm{AF}}}{M_{\mathrm{FM}} t_{\mathrm{FM}}},
$$

also $\mu_{0} H_{\mathrm{EB}}(n=1)$ follows a $1 / t_{\mathrm{FM}}$ dependence. Equation (1) relates the EB field to a phenomenological coupling $J$ between the FM and AF interface magnetization $S_{\mathrm{FM}}$ and $S_{\mathrm{AF}}$, and the saturation magnetization $M_{\mathrm{FM}}$ of the FM film of thickness $t_{\mathrm{FM}}$. Therefore, scaling according to $\mu_{0} H_{\mathrm{EB}}(n=1)$ $\times t_{\mathrm{FM}}$ vs $T$ as shown in Fig. 7(b) is expected. Since each individual data set follows empirically a linear $T$ dependence, data collapse takes place on a virtually linear master curve. The line shows a best fit to the scaled data $\mu_{0} H_{\mathrm{EB}}(n=1) \times t_{\mathrm{FM}}$ vs $T$ with slope $a=-0.0387 \mathrm{~T} \mathrm{~nm} / \mathrm{K}$ and ordinate intercept $b=3.3697 \mathrm{~T} \mathrm{~nm}$. Its extrapolation towards $\mu_{0} H_{\mathrm{EB}}(n=1) \times t_{\mathrm{FM}}=0$ determines the blocking temperature $T_{B}=96.8 \mathrm{~K}$.

Figure 8(a) shows $\mu_{0} H_{\mathrm{EB}}(n=1)$ vs $t_{\mathrm{FM}}$ for $T=20$ (squares), 27 (circles), 35 (up triangles), 43 (down triangles), 50 (diamonds), 57 (left triangles), 65 (right triangles), and $70 \mathrm{~K}$ (hexagons), respectively. As expected, the individual data sets follow the $1 / t_{\mathrm{FM}}$ dependence of Eq. (1). The lines
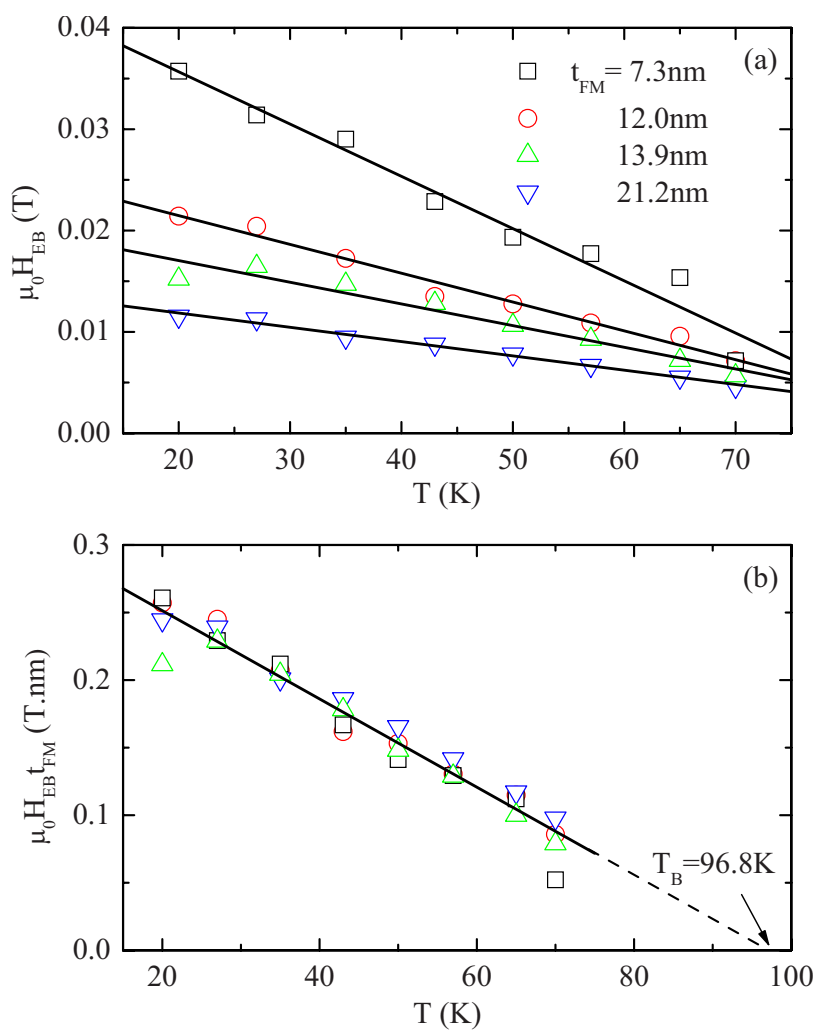

FIG. 7. (Color online) (a) Variation of exchange bias $\mu_{0} H_{\mathrm{EB}}$ vs $T$ for Co thickness values $7.3 \mathrm{~nm}$ (squares), $12.0 \mathrm{~nm}$ (circles), $13.9 \mathrm{~nm}$ (up triangles), and $21.2 \mathrm{~nm}$ (down triangles). The lines are the linear fits. (b) The master line $\mu_{0} H_{\mathrm{EB}} t_{\mathrm{FM}}$ vs $T$ with corresponding scaled data and the blocking temperature $T_{B}=96.8 \mathrm{~K}$ marked by an arrow at the intercept of the master line with the $T$ axis.

are best fits to Eq. (1), where $P_{1}=-J S_{\mathrm{FM}} S_{A F} / M_{\mathrm{FM}}$ becomes the temperature-dependent fitting parameter for each data set. Recalling the fitting parameters $a$ and $b$ of the linear master curve of Fig. 7(b) we create a data collapse according to the scaling $\mu_{0} H_{\mathrm{EB}}(n=1) /(a T+b)$ vs $t_{\mathrm{FM}}$. Figure $8(\mathrm{~b})$ shows the result of this scaling which reflects the $1 / t_{\mathrm{FM}}$ dependence of the individual data sets. The master curve of the scaled $\mu_{0} H_{\mathrm{EB}}(n=1) /(a T+b)$ vs $t_{\mathrm{FM}}$ data is again obtained by a best fit to $g\left(t_{\mathrm{FM}}\right)=g_{0} / t_{\mathrm{FM}}$ where the unit free fitting parameter reads $g_{0}=0.1051 \pm 0.0025$.

As outlined in Sec. II, the nominal thicknesses $t_{\mathrm{FM}}$ suffer from experimental uncertainties $\Delta t_{\mathrm{FM}} / t_{\mathrm{FM}}$ of up to $23 \%$. However, the master curve $g\left(t_{\mathrm{FM}}\right)$ of Fig. 8(b) allows for the determination of scaled thicknesses $t_{\mathrm{FM}}^{\text {scaled }}$. They are to a large extent free from the experimental errors originating from $\Delta x$ uncertainties. Considering the quality of our Kerr loops it is reasonable that the statistical deviations of the data points from the master curve originate from errors in $t_{\mathrm{FM}}$ while errors in the EB fields of the first loops are negligible. Under this consideration $t_{\mathrm{FM}}^{\text {scaled }}$ is obtained from the relation $g_{0} / t_{\mathrm{FM}}^{\text {scaled }}=\mu_{0} H_{\mathrm{EB}}\left(n=1, t_{\mathrm{FM}}\right) /(a T+b)$. Geometrically, this correction procedure describes a shift of the data points along the $t_{\mathrm{FM}}$ axis onto the master curve. This procedure is indicated in Fig. 8(b) by horizontal arrows for two exemplary data points. The resulting relative corrections $\mid t_{\mathrm{FM}}^{\text {scaled }}$ 

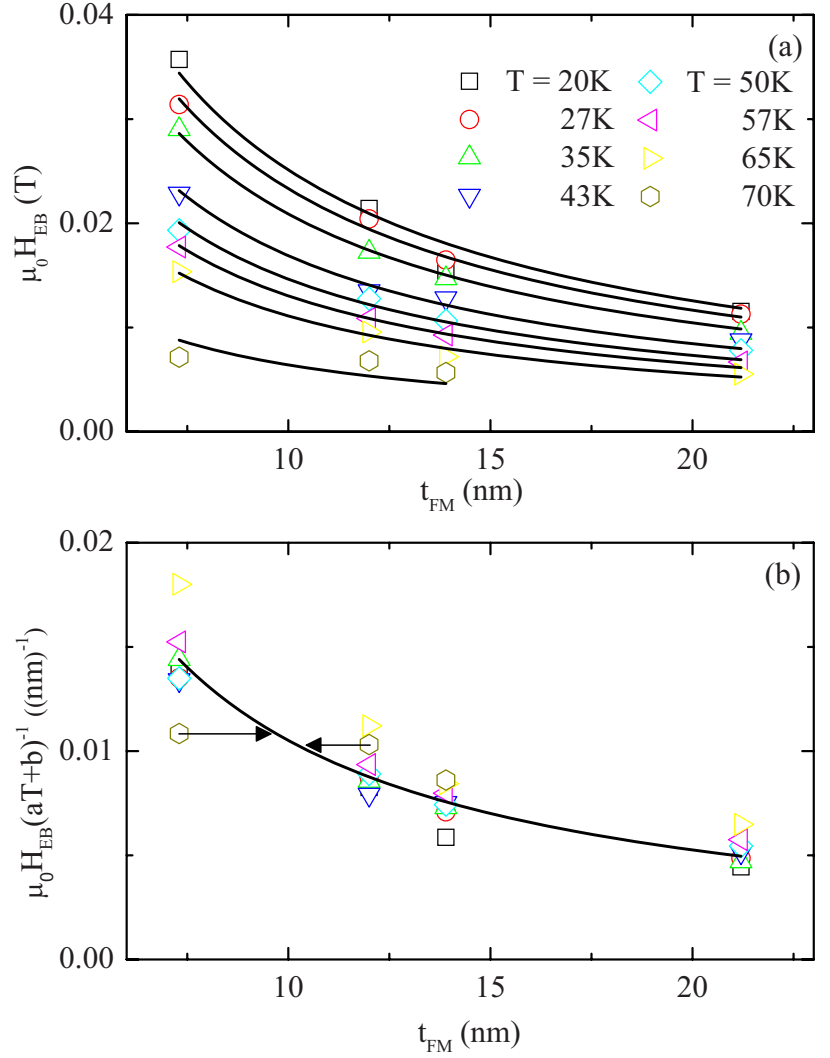

FIG. 8. (Color online) (a) Variation of exchange bias $\mu_{0} H_{\mathrm{EB}}$ vs ferromagnet thickness $t_{\mathrm{FM}}$ at different temperatures. The lines are best fits to Eq. (1). (b) Scaled data $\mu_{0} H_{\mathrm{EB}} \times(a T+b)^{-1}$ vs $t_{\mathrm{FM}}$ (for details regarding $a$ and $b$ see text). The master curve is represented by a best fit (line) of a Meiklejon-Bean-type formula to the scaled data. Arrows provide a geometrical interpretation of the thickness correction assigning scaled thickness values to the nominal thicknesses.

$-t_{\mathrm{FM}} \mid / t_{\mathrm{FM}}$ are within the expected maximum error $\Delta t / t$ $\approx \Delta x / w=23 \%$ associated with the $\Delta x$ uncertainties.

Figure 9 shows a three-dimensional plot of $\mu_{0} H_{\mathrm{EB}}(n$ $=1)$ vs $\left(t_{\mathrm{FM}}^{\text {scaled }}, T\right)$ for all scaled thicknesses and temperatures. All data points fall on a smoothly curved surface indicating that $\mu_{0} H_{\mathrm{EB}}(n=1)$ decreases with increasing temperature as well as FM thickness. The smoothness of the interpolating surface indicates that in fact the thickness correction effectively eliminates the errors in the nominal thicknesses $t_{\mathrm{FM}}$. Note, that due to the scaling procedure $t_{\mathrm{FM}} \rightarrow t_{\mathrm{FM}}^{\text {scaled }}$ the $\mu_{0} H_{\mathrm{EB}}(n=1)$-data points do not follow isothickness lines.

Figure 10 shows a similar three-dimensional plot for the loop width $\mu_{0} H_{c}(n=1)$ vs $\left(t_{\mathrm{FM}}^{\text {scaled }}, T\right)$, of the first loop of a respective training sequence for all scaled thicknesses and temperatures. The loop width or coercivity is known to increase with decreasing temperature below the EB blocking temperature $T_{B}$. Qualitatively this behavior can be understood due to the drag effect the FM interface spins experience on magnetization reversal. In addition, Fig. 10 shows an increase of the coercivity with decreasing FM thickness. Recently, Scholten et al. provided a mean-field solution for the coercivity change in EB heterolayers. ${ }^{49}$ It reads

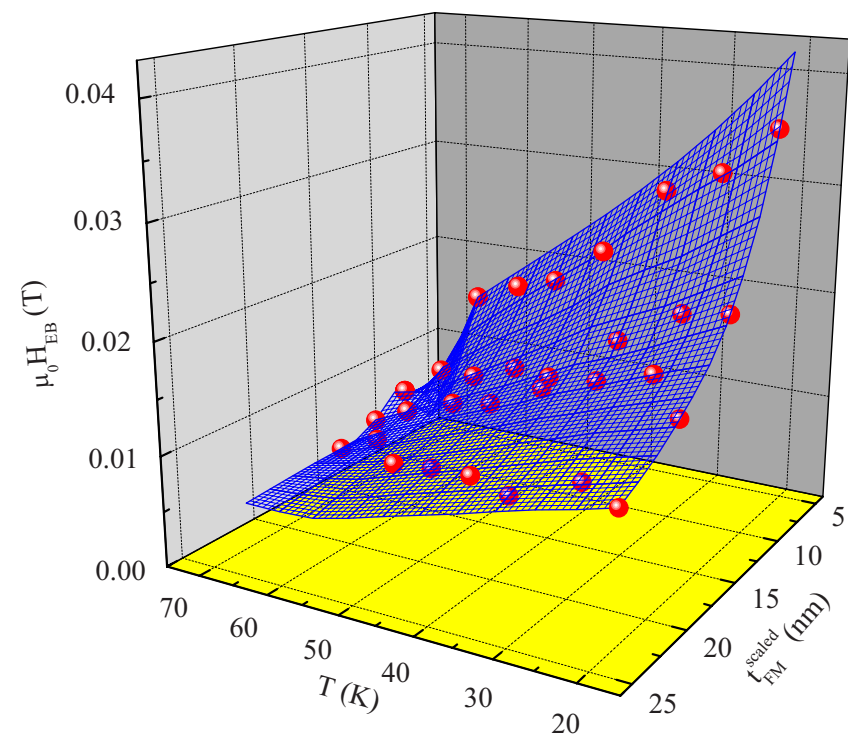

FIG. 9. (Color online) 3D plot illustrating the exchange bias $\mu_{0} H_{\mathrm{EB}}$ vs $\left(t_{\mathrm{FM}}^{\text {scaled }}, T\right)$. The spheres are the experimental data and the interpolating grid results from Renka-Cline gridding algorithm.

$$
\mu_{0} H_{c}\left(t_{\mathrm{FM}}\right)=\frac{\mu_{0} H_{c}^{\infty}+J^{2} \chi / t_{\mathrm{FM}}}{1+J \chi / t_{\mathrm{FM}}}
$$

where $\mu_{0} H_{c}^{\infty}=\mu_{0} H_{c}\left(t_{\mathrm{FM}} \rightarrow \infty\right)$ is the FM bulk coercivity and $\chi$ is the temperature-dependent magnetic susceptibility of the $\mathrm{AF}$ layer at the interface. Individual best fits of Eq. (2) to $\mu_{0} H_{c}$ vs $t_{\mathrm{FM}}^{\text {scaled }}$ at constant temperature (not shown) indicate $J \chi / t_{\mathrm{FM}} \ll 1$ and $\mu_{0} H_{c}^{\infty} \ll \mu_{0} H_{c}\left(t_{\mathrm{FM}}\right)$ for all studied thicknesses. Therefore an approximate $1 / t_{\mathrm{FM}}$ behavior is expected not only for $\mu_{0} H_{\mathrm{EB}}(n=1)$ but also for $\mu_{0} H_{c}(n=1)$ vs $T$. The latter is consistent with the intuitive picture that the coerciv-

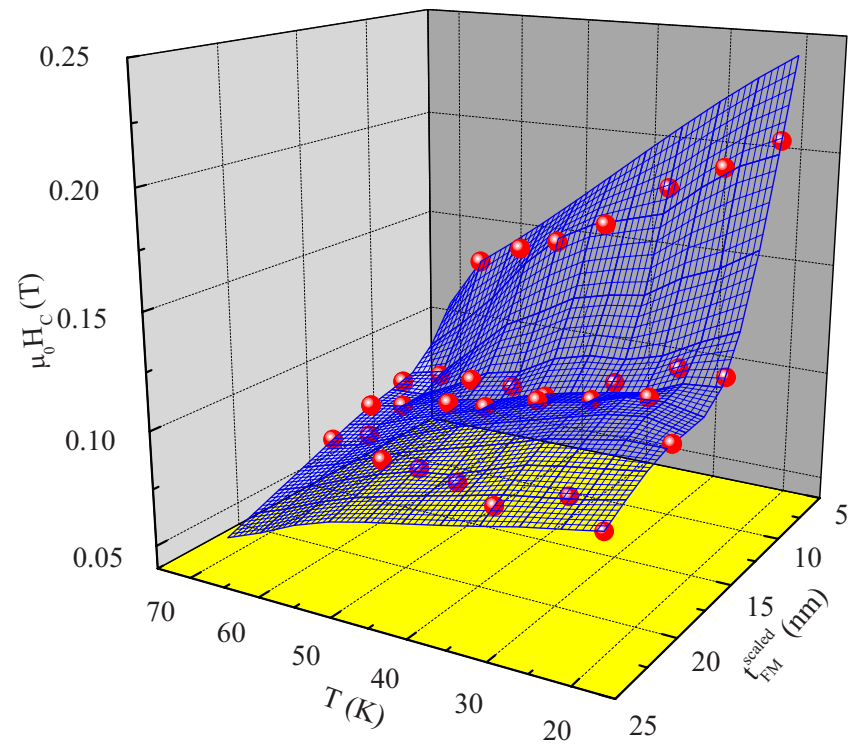

FIG. 10. (Color online) 3D plot illustrating the loop width $\mu_{0} H_{\mathrm{C}}$ vs $\left(t_{\mathrm{FM}}^{\mathrm{scaled}}, T\right)$. The spheres are the experimental data and the interpolating grid results from Renka-Cline gridding algorithm. 
ity enhancement in EB system is an interface effect. $1 / t_{\mathrm{FM}}$ dependence and more general $\left(1 / t_{\mathrm{FM}}\right)^{\alpha}$ behavior of $\mu_{0} H_{c}\left(t_{\mathrm{FM}}\right)$ has been observed in various EB systems. ${ }^{50,12,51}$

From Eq. (2) and its successful application to the $\mu_{0} H_{c}$ vs $\left(t_{\mathrm{FM}}^{\text {scaled }}, T\right)$ data it is apparent that the thickness dependence of the FM loop width is related to the AF interface susceptibility. Hence, one might expect that the AF interface magnetization and, with it, the EB training effect depends on the FM film thickness in a nontrivial $1 / t_{\mathrm{FM}}^{\text {scaled }}$ manner. Subsequently we evidence, however, that the training effect in our $\mathrm{Co} / \mathrm{CoO}$ samples reflects only the explicit $1 / t_{\mathrm{FM}}^{\text {scaled }}$ dependence of Eq. (1) implying that $S_{\mathrm{AF}}$ vs $n$ does not or only insignificantly depend on $t_{\mathrm{FM}}^{\text {scaled }}$. We evidence this statement with the help of the recently introduced implicit sequence for the EB training effect ${ }^{39,40,3}$

$$
\mu_{0}\left[H_{\mathrm{EB}}(n+1)-H_{\mathrm{EB}}(n)\right]=-\gamma\left\{\mu_{0}\left[H_{\mathrm{EB}}(n)-H_{\mathrm{EB}}^{e}\right]\right\}^{3},
$$

where fitting parameters $\mu_{0} H_{\mathrm{EB}}^{e}$ and $\gamma$ describe $\mu_{0} H_{\mathrm{EB}}$ vs $n$ in the limit $n \rightarrow \infty$ and the characteristic decay rate of the training behavior, respectively. While Eq. (3) has mainly been applied to cases where the $\mu_{0} H_{\mathrm{EB}}$ shows a gradual $n$ dependence, $3,18,19,24,39,40$ it also has the potential to reproduce steplike characteristics where training takes place only between the first and second loop. This is in strong contrast to recent interpretations ${ }^{52}$ of Eq. (3). It is straightforward to show, that

$$
\gamma=\frac{1}{\left[\mu_{0} H_{\mathrm{EB}}(n=1)-\mu_{0} H_{\mathrm{EB}}^{e}\right]^{2}}
$$

gives rise to pure steplike characteristics of $\mu_{0} H_{\mathrm{EB}}$ vs $n$. Defining a steepness parameter $C$ as $C=\left[H_{\mathrm{EB}}(n=1)-H_{\mathrm{EB}}(n\right.$ $=2)] /\left[H_{\mathrm{EB}}(n=1)-H_{\mathrm{EB}}^{e}\right]$ which quantifies the characteristics of the training behavior one can show $\gamma=C /\left[\mu_{0} H_{\mathrm{EB}}(n=1)\right.$ $\left.-\mu_{0} H_{\mathrm{EB}}^{e}\right]^{2}$ where $0 \leqslant C \leqslant 1$. $C=1$ resembles steplike behavior while $C<1$ gives rise to gradual behavior of $\mu_{0} H_{\mathrm{EB}}$ vs $n$ for $n>2$. In our case $C$ is typically $\approx 0.9$.

Equation (3) has been best fitted to all training data sets. Figures 6(a)-6(d) shows four typical examples of the fitting results (squares) using the equilibrium EB field $\mu_{0} H_{\mathrm{EB}}^{e}$ and $\gamma$ as fitting parameters.

Figure 11 shows a three-dimensional plot of the crucial fitting parameter $\gamma$ vs $\left(t_{\mathrm{FM}}^{\text {scaled }}, T\right)$. Recently we derived a mean-field expression for the temperature dependence of $\gamma^{40}$ In accordance with this result the isothickness lines $\gamma$ vs $T$ show an increase of $\gamma$ with increasing temperature. The isotherms follow a $\gamma \propto\left(t_{\mathrm{FM}}^{\text {scaled }}\right)^{2}$ behavior suggesting a scaling plot $\gamma /\left(t_{\mathrm{FM}}^{\text {scaled }}\right)^{2}$ vs $T$. Figure 12 displays this scaling plot as the essence of our study. Within the error bars perfect data collapse onto a master curve is achieved. The line is a single parameter fit using the fixed blocking temperature $T_{B}$ $=96.8 \mathrm{~K}$ in the mean-field expression of Ref. 40 .

The fact that data collapse is achieved on the basis $\gamma$ $\propto\left(t_{\mathrm{FM}}^{\text {scaled }}\right)^{2}$ implies $\mu_{0} H_{\mathrm{EB}}(n) \propto 1 / t_{\mathrm{FM}}^{\text {scaled }}$ and $S_{\mathrm{AF}}(n)$ independent of $t_{\mathrm{FM}}^{\text {scaled }}$. This can be seen when generalizing Eq. (1) for all loops in a training sequence according to $\mu_{0} H_{\mathrm{FM}}(n)$ $=-J S_{\mathrm{FM}} S_{\mathrm{AF}}(n) /\left(M_{\mathrm{FM}} t_{\mathrm{FM}}^{\text {scaled }}\right)$ and substituting it into Eq. (3). Some rearrangements yield

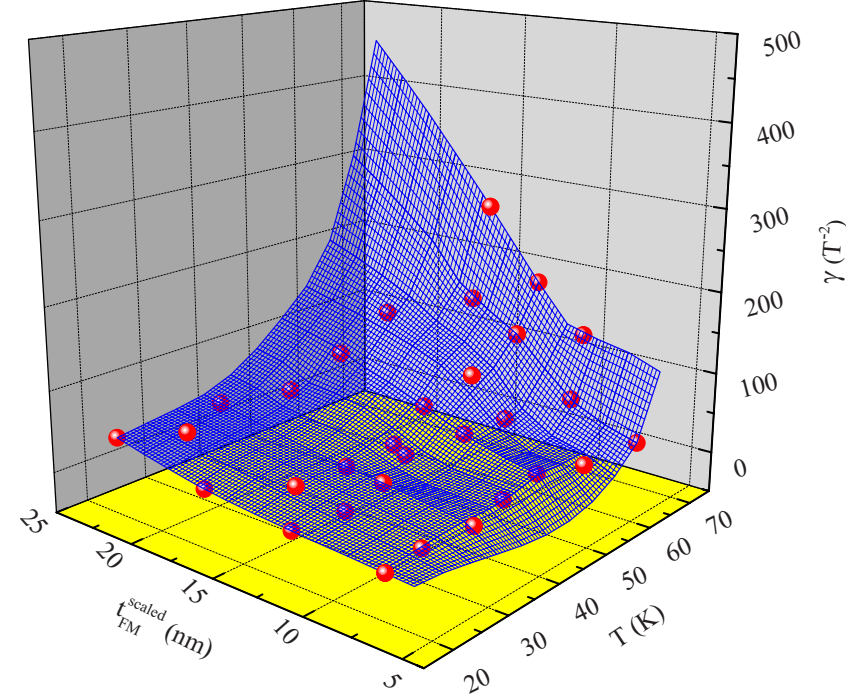

FIG. 11. (Color online) 3D plot illustrating fitting parameter $\gamma$ vs $\left(t_{\mathrm{FM}}^{\text {scaled }}, T\right)$. The $\gamma$ values are obtained from best fits of the training data to Eq. (3). The spheres are the experimental data and the simulated grid results from Renka-Cline gridding algorithm.

$$
\gamma=\left(t_{\mathrm{FM}}^{\text {scaled }}\right)^{2}\left(\frac{M_{\mathrm{FM}}}{\mu_{0} J S_{\mathrm{FM}}}\right)^{2} \frac{S_{\mathrm{AF}}(n+1)-S_{\mathrm{AF}}(n)}{\left[S_{\mathrm{AF}}^{e}-S_{\mathrm{AF}}(n)\right]^{3}} \propto\left(t_{\mathrm{FM}}^{\text {scaled }}\right)^{2},
$$

where $S_{\mathrm{AF}}^{e}$ is the quasiequilibrium $\mathrm{AF}$ interface magnetization achieved in the limit $n \rightarrow \infty$. Note, that $\gamma \propto\left(t_{\mathrm{FM}}^{\text {scaled }}\right)^{2}$ is a direct consequence of $S_{\mathrm{AF}}(n)$ being independent of $t_{\mathrm{FM}}^{\text {scaled }}$. Note in addition that the $\left(t_{\mathrm{FM}}^{\text {scaled }}\right)^{2}$ scaling of $\gamma$ is strong evidence for the validity of the underlying theoretical approach.

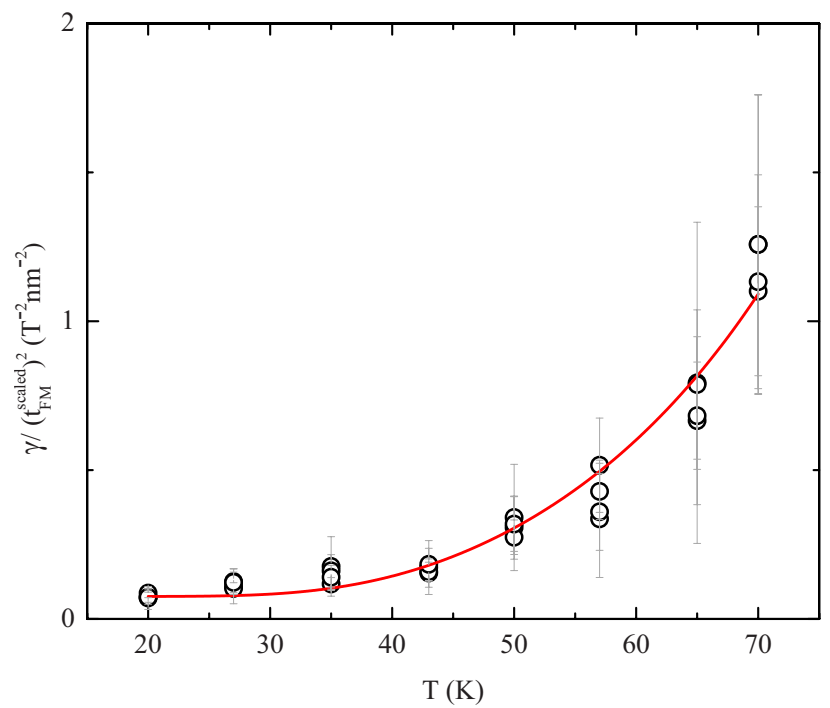

FIG. 12. (Color online) Scaling plot $\gamma /\left(t_{\mathrm{FM}}^{\text {scaled }}\right)^{2}$ vs $T$. The line represents a best fit of the mean-field result for the temperature dependence of $\gamma$ (see Ref. 40) to the data (circles). The error bars reflect the maximum deviations of $\gamma$ related to thickness fluctuations. 
The latter is based on triggered relaxation of the pinning layer towards quasiequilibrium. The dynamics of this triggered relaxation process is controlled via a discretized Landau-Khalatnikov equation involving the free energy difference $\Delta F \propto\left[S_{\mathrm{AF}}(n)-S_{\mathrm{AF}}^{e}\right]^{4}$ between $S_{\mathrm{AF}}^{e}$ and $S_{\mathrm{AF}}(n)$ for a given loop $n \cdot{ }^{39,40}$ The functional form of the free energy involving the fourth power in the difference of the interface magnetizations gives rise to the functional form of the implicit Eq. (3). Note, that only the cubic term on the right side of the expression of Eq. (3) provides $\gamma \propto\left(t_{\mathrm{FM}}^{\text {scaled }}\right)^{2}$. This is overwhelming evidence for the underlying structure of the free energy.

\section{CONCLUSIONS}

We studied scaling behavior of the exchange bias training effect on the ferromagnetic film thickness and temperature in a single $\mathrm{CoO} / \mathrm{Co}$-wedge heterostructure. The study is partially motivated by the observed entanglement between the coercivity of the ferromagnetic film, its thickness dependence and its relation with the antiferromagnetic interface susceptibility. A possible change of the retroactivity of the ferromagnet onto the antiferromagnetic interface magnetiza- tion with changing ferromagnetic film thickness leaves, however, no fingerprint in the exchange bias training effect. This is evidenced by a detailed scaling analysis showing that each individual exchange bias field within a training sequence resembles the same well-know inverse thickness dependence on the ferromagnetic film thickness. This finding implies that the evolution of the antiferromagnetic interface magnetization is independent of the ferromagnetic film thickness. Nevertheless, training of the absolute exchange bias fields shows a ferromagnetic thickness dependence entering the corresponding theory in a nontrivial manner. Scaling behavior of the crucial fitting parameter involved in the latter provides unprecedented evidence for the underlying phenomenological approach based on discretized Landau-Khalatnikov dynamics.

\section{ACKNOWLEDGMENTS}

We thank Brian Jones and Tathagata Mukherjee for technical support. This research work is supported by NSF through Grant No. DMR-0547887, the Nebraska Research Initiative (NRI), and by the MRSEC Program of the NSF (Grant No. DMR-0213808). *cbinek2@unl.edu

${ }^{1}$ Th. Mühge, N. N. Garif'yanov, Yu. V. Goryunov, G. G. Khaliullin, L. R. Tagirov, K. Westerholt, I. A. Garifullin, and H. Zabel, Phys. Rev. Lett. 77, 1857 (1996).

${ }^{2}$ Chun-Gang Duan, S. S. Jaswal, and E. Y. Tsymbal, Phys. Rev. Lett. 97, 047201 (2006).

${ }^{3}$ Ch. Binek, S. Polisetty, Xi He, and A. Berger, Phys. Rev. Lett. 96, 067201 (2006).

${ }^{4}$ I. Žutić, J. Fabian, and S. Das Sarma, Rev. Mod. Phys. 76, 323 (2004).

${ }^{5}$ W. H. Meiklejohn and C. P. Bean, Phys. Rev. 102, 1413 (1956).

${ }^{6}$ W. H. Meiklejohn and C. P. Bean, Phys. Rev. 105, 904 (1956).

${ }^{7}$ J. Nogués and I. K. Schuller, J. Magn. Magn. Mater. 192, 203 (1999).

${ }^{8}$ A. Berkowitz and K. Takano, J. Magn. Magn. Mater. 200, 552 (1999).

${ }^{9}$ R. L. Stamps, J. Phys. D 33, R247 (2000).

${ }^{10}$ M. Kiwi, J. Magn. Magn. Mater. 234, 584 (2001).

${ }^{11}$ I. Roshchin, O. Petracic, R. Morales, Z.-P. Li, X. Batlle, and I. K. Schuller, Europhys. Lett. 71, 297 (2005).

${ }^{12}$ C. Leighton, J. Nogués, B. J. Jönsson-Åkerman, and I. K. Schuller, Phys. Rev. Lett. 84, 3466 (2000).

${ }^{13}$ S. Maat, K. Takano, S. S. P. Parkin, and E. E. Fullerton, Phys. Rev. Lett. 87, 087202 (2001).

${ }^{14}$ D. Paccard, C. Schlenker, O. Massenet, R. Montmory, and A. Yelon, Phys. Status Solidi 16, 301 (1966).

${ }^{15}$ C. Schlenker, S. S. P. Parkin, J. C. Scott, and K. Howard, J. Magn. Magn. Mater. 54, 801 (1986).

${ }^{16}$ K. Zhang, T. Zhao, and M. Fujiwara, J. Appl. Phys. 89, 6910 (2001).

${ }^{17}$ S. G. te Velthuis, A. Berger, G. P. Felcher, B. Hill, and E. Dahlberg, J. Appl. Phys. 87, 5046 (2000).
${ }^{18}$ Heiwan Xi, R. M. White, S. Mao, Z. Gao, Z. Yang, and E. Murdock, Phys. Rev. B 64, 184416 (2001).

${ }^{19}$ S. Sahoo, S. Polisetty, Ch. Binek, and A. Berger, J. Appl. Phys. 101, 053902 (2007).

${ }^{20}$ Haiwen Xi, S. Franzen, S. Mao, and R. M. White, Phys. Rev. B 75, 014434 (2007).

${ }^{21}$ A. Hoffmann, Phys. Rev. Lett. 93, 097203 (2004).

${ }^{22}$ V. Skumryev, S. Stoyanov, Y. Zhang, G. Hadjipanayis, D. Givord, and J. Nogués, Nature (London) 423, 850 (2003).

${ }^{23}$ A. Hochstrat, Ch. Binek, and W. Kleemann, Phys. Rev. B 66, 092409 (2002).

${ }^{24}$ D. Niebieskikwiat and M. B. Salamon, Phys. Rev. B 72, 174422 (2005).

${ }^{25}$ Z.-P. Li, O. Petracic, J. Eisenmenger, and I. K. Schuller, Appl. Phys. Lett. 86, 072501 (2005).

${ }^{26}$ J. Eisenmenger, Z.-P. Li, W. A. A. Macedo, and I. K. Schuller, Phys. Rev. Lett. 94, 057203 (2005).

${ }^{27}$ V. Baltz, J. Sort, S. Landis, B. Rodmacq, and B. Dieny, Phys. Rev. Lett. 94, 117201 (2005).

${ }^{28}$ G. Malinowski, M. Albrecht, I. L. Guhr, J. M. D. Coey, and S. van Dijken, Phys. Rev. B 75, 012413 (2007).

${ }^{29}$ A. N. Dobrynin, D. N. Ievlev, K. Temst, P. Lievens, J. Margueritat, J. Gonzalo, C. N. Afonso, S. Q. Zhou, A. Vantomme, E. Piscopiello, and G. Van Tendeloo, Appl. Phys. Lett. 87, 012501 (2005)

${ }^{30} \mathrm{Ch}$. Binek, A. Hochstrat, and W. Kleemann, J. Magn. Magn. Mater. 234, 353 (2001).

${ }^{31}$ S. S. P. Parkin and V. Speriosu, Springer Proc. Phys. 50, 110 (1990).

${ }^{32}$ K. Seu, H. Huang, J. L. Showman, H. Showman, W. F. Egelhoff, Jr., and A. Reilly, J. Appl. Phys. 93, 6611 (2003).

${ }^{33}$ F. Radu, M. Etzkorn, R. Siebrecht, T. Schmitte, K. Westerholt, 
and H. Zabel, Phys. Rev. B 67, 134409 (2003).

${ }^{34}$ C. Leighton, M. R. Fitzsimmons, A. Hoffmann, J. Dura, C. F. Majkrzak, M. S. Lund, and Ivan K. Schuller, Phys. Rev. B 65, 064403 (2002).

${ }^{35}$ S. Brems, D. Buntinx, K. Temst, C. Van Haesendonck, F. Radu, and H. Zabel, Phys. Rev. Lett. 95, 157202 (2005).

${ }^{36} \mathrm{~S}$. Brems, K. Temst, and C. Van Haesendonck, Phys. Rev. Lett. 99, 067201 (2007).

${ }^{37}$ Ch. Binek, Ising-type Antiferromagnets: Model Systems in Statistical Physics and the Magnetism of Exchange Bias, Springer Tracts in Modern Physics Vol. 196 (Springer, Berlin, 2003).

${ }^{38} \mathrm{Ch}$. Binek, A. Hochstrat, and W. Kleemann, Phys. Status Solidi B 189, 575 (2002).

${ }^{39}$ Ch. Binek, Phys. Rev. B 70, 014421 (2004).

${ }^{40}$ Ch. Binek, Xi He, and S. Polisetty, Phys. Rev. B 72, 054408 (2005).

${ }^{41}$ L. Smardz, Phys. Status Solidi A 181, R7 (2000).

${ }^{42}$ F. Celestini and F. Mortessagne (unpublished).

${ }^{43}$ M. S. Lund, W. A. A. Macedo, Kai Liu, J. Nogués, Ivan K.
Schuller, and C. Leighton, Phys. Rev. B 66, 054422 (2002).

${ }^{44}$ A. Stierle, A. Abromeit, N. Metoki, and H. Zabel, J. Appl. Phys. 73, 4808 (1993).

${ }^{45}$ J. Scheffler, S. Polisetty, S. Sahoo, and Ch. Binek (unpublished).

${ }^{46}$ Th. Zeidler, F. Schreiber, H. Zabel, W. Donner, and N. Metoki, Phys. Rev. B 53, 3256 (1996).

${ }^{47}$ R. Allenspach, M. Stampanoni, and A. Bischof, Phys. Rev. Lett. 65, 3344 (1990).

${ }^{48}$ H. Shi and D. Lederman, J. Appl. Phys. 87, 6095 (2000).

${ }^{49}$ G. Scholten, K. D. Usadel, and U. Nowak, Phys. Rev. B 71, 064413 (2005)

${ }^{50}$ T. C. Schulthess and W. H. Butler, J. Appl. Phys. 85, 5510 (1999).

${ }^{51}$ M. D. Stiles and R. D. McMichael, Phys. Rev. B 63, 064405 (2001).

${ }^{52}$ A. Paul, T. Brückel, E. Kentzinger, and U. Rücker, J. Phys.: Condens. Matter 19, 086229 (2007). 